\title{
SEMISIMPLE REPRESENTATIONS OF QUIVERS
}

\author{
LIEVEN LE BRUYN AND CLAUDIO PROCESI
}

\begin{abstract}
We discuss the invariant theory of the variety of representations of a quiver and present generators and relations.

We connect this theory of algebras with a trace satisfying a formal CayleyHamilton identity
\end{abstract}

\section{INTRODUCTION}

After the work of P. Gabriel [Ga], it became clear that a wide variety of problems from linear algebra could be formulated and studied in a uniform way in the context of representations of quivers. We will briefly recall the setting:

Throughout this paper, we work over an algebraically closed field of characteristic zero and call it $\mathbf{C}$. A quiver $Q$ is a fourtuple $\left(Q_{0}, Q_{1}, t, h\right)$ consisting of a finite set $Q_{0}=\{1, \ldots, n\}$ of vertices, a finite set $Q_{1}=\{\phi \in \Phi\}$ of arrows between these vertices and two maps $t, h: Q_{1} \rightarrow Q_{0}$ assigning to an arrow $\phi$ its tail $t(\phi)$ and its head $h(\phi)$ respectively. Note that we do not exclude loops or multiple arrows. However, we will always assume that the underlying graph of the quiver is connected.

A representation $V$ of a quiver $Q$ is a family $\left\{V(i): i \in Q_{0}\right\}$ of finite dimensional vector spaces over $\mathbf{C}$ together with a family of linear maps $\{V(\phi): V(t(\phi))$ $\left.\rightarrow V(h(\phi)) ; \phi \in Q_{1}\right\}$. The $n$-tuple of integers $\operatorname{dim}(V)=(\operatorname{dim}(V(i)))_{i} \in$ $\mathbf{N}^{n}$ is called the dimension vector of the representation $V$. A morphism between two representations $f: V \rightarrow W$ is a family of linear morphisms $\left\{f(i): V(i) \rightarrow W(i) ; i \in Q_{0}\right\}$ such that for all arrows $\phi \in Q_{1}$ we have that $W(\phi) \circ f(t(\phi))=f(h(\phi)) \circ V(\phi)$. A morphism $f$ is an isomorphism if all the components $f(i)$ are isomorphisms.

For a fixed dimension vector $\alpha=(\alpha(1), \ldots, \alpha(n)) \in \mathbf{N}^{n}$ we define the representation space $R(Q, \alpha)$ of the quiver $Q$ to be the set of all representations $V$ of $Q$ such that $V(i)=\mathbf{C}^{\alpha(i)}$ for all $i \in Q_{0}$. Because $V \in R(Q, \alpha)$ is completely determined by the linear morphisms $V(\phi)$ we have that

$$
R(Q, \alpha)=\bigoplus_{\phi \in Q_{1}} \operatorname{Hom}_{\mathbf{C}}\left(\mathbf{C}^{\alpha(t(\phi))}, \mathbf{C}^{\alpha(h(\phi))}\right)=\bigoplus_{\phi \in Q_{1}} M_{\phi}(\mathbf{C})
$$

Received by the editors February 1, 1988.

1980 Mathematics Subject Classification (1985 Revision). Primary 16A38, 14L25. 
where for each $\phi \in Q_{1}$ we denote by $M_{\phi}(\mathbf{C})$ the vectorspace of all $\alpha(h(\phi))$ by $\alpha(t(\phi))$ matrices with entries in $\mathbf{C}$.

We will consider the vectorspace $R(Q, \alpha)$ as an affine variety with coordinate ring $\mathbf{C}[Q, \alpha]$ and functionfield $\mathbf{C}(Q, \alpha)$. There is a canonical action of the linear reductive group

$$
G L(\alpha)=\prod_{i=1}^{n} G L_{\alpha(i)}(\mathbf{C})
$$

on the representation space $R(Q, \alpha)$ determined for all representations $V \in$ $R(Q, \alpha)$ and all group elements $g=(g(1), \ldots, g(n)) \in G L(\alpha)$ by the rule

$$
(g \cdot V)(\phi)=g(h(\phi)) V(\phi) g(t(\phi))^{-1} .
$$

It is clear that the $G L(\alpha)$-orbits in $R(Q, \alpha)$ are precisely the isomorphism classes of representations.

As we will recall in the next section, every representation $V \in R(Q, \alpha)$ can be written (but not necessarily uniquely) as $V=V_{s}+V_{n}$ where $V_{s}$ is a semisimple representation and $V_{n}$ is such that the zero representation lies in the closure of the orbit of $V_{n}$ under the stabilizer subgroup of $V_{s}$. We then call $V=V_{s}+V_{n}$ a Jordan decomposition of the representation $V$. Therefore the classification of the orbit structure of $G L(\alpha)$ on $R(Q, \alpha)$ can be divided up in two subproblems:

(I): the study of all semisimple representations of $Q$ and

(II): the study of nilpotent representations of $Q$ with respect to certain linear reductive subgroups of $G L(\alpha)$.

In this paper we aim to apply the étale slice machinery of D. Luna [Lu1] in order to get a fairly complete answer to problem (I). Concerning problem (II) we will determine the finitely many linear reductive subgroups of $G L(\alpha)$ which occur as the stabilizer subgroup of a semisimple representation.

Since semisimple representations are precisely those representations $V \in$ $R(Q, \alpha)$ such that the corresponding orbit $G L(\alpha) \cdot V$ is closed, it follows from Mumford's theory [Mu] that their isomorphism classes are parametrized by the quotient variety $V(Q, \alpha)=R(Q, \alpha) / G L(\alpha)$. In $\S 3$ we will show that the coordinate ring of this variety (the ring of polynomial invariants) is generated by traces of oriented cycles in the quiver $Q$ and we give a bound on the length of the cycles required.

In $\S 4$ we will see that the quotient variety $V(Q, \alpha)$ admits a finite stratification into locally closed smooth irreducible subvarieties corresponding to the different types of semisimple decompositions of dimension vector $\alpha$. Moreover, one stratum lies in the closure of another if the corresponding representations are deformations.

Using these two result we will determine in $\S 5$ all dimension vectors which occur as the dimension vector of a simple representation. This problem can be viewed analogous to (but much easier than) the corresponding problem for indecomposable representations which has been solved by V. Kac [Ka]. Our description is expressed in terms of the bilinear Ringel form $R(\alpha, \beta)$ whose 
symmetrization is the Tits quadratic form corresponding to the root system of the quiver $Q$. Therefore, we obtain a purely combinatorial method to determine all types of semisimple decompositions of dimension vector $\alpha$ and hence of all the linear reductive subgroups which occur in the problem (II) mentioned above.

In $\S 6$ we will concentrate on the analytic local structure of the quotient variety $V(Q, \alpha)$. For a given semisimple representation type $\tau=\left(e_{1}, \beta_{1} ; \ldots ; e_{l}, \beta_{l}\right)$ we will construct a new quiver $Q_{\tau}$ with vertex set $\{1, \ldots, l\}$ such that there are $\delta_{i j}-R\left(\beta_{i}, \beta_{j}\right)$ arrows pointing from $i$ to $j$ and we consider a new dimension vector $\alpha_{\tau}=\left(e_{1}, \ldots, e_{l}\right)$. We then prove that there is an étale morphism from a neighborhood of the origin in the quotient variety $V\left(Q_{\tau}, \alpha_{\tau}\right)$ to a neighborhood of any point $\xi$ in $V(Q, \alpha)$ of representation type $\tau$. This result simplifies the study in all points except for an $m$-dimensional subspace where $m$ is the number of loops in the quiver $Q$.

In the last section we will compute the Krull dimension of the quotient variety $V(Q, \alpha)$ and determine its singular locus. Both answers are given in terms of the generic semisimple representation type (i.e. the unique open stratum in the stratification of $\S 4$ ) and we will present a combinatorial method to determine this generic type. Note that the corresponding problem for arbitrary representations is still open, although A. Schofield [Sc] has recently obtained some encouraging results.

Acknowledgment. We thank C. M. Ringel for his stimulating interest in the topic of this paper and for his generous help in proving the results of $\S 5$.

\section{THE ÉTALE SLICE MACHINERY}

In this section we will briefly recall some of the general results due to D. Luna [Lu1, Lu2] which will be used throughout this paper.

Let $G$ be a linear reductive group which acts linearly on a finite dimensional vectorspace $X$ over $\mathbf{C}$. As we mentioned in the introduction, there exist Jordan decomposition(s) for any element in $X$. An element $x \in X$ is said to be semisimple (resp. nilpotent) with respect to $G$ iff the orbit $G \cdot x$ is closed (resp. $0 \in \overline{G \cdot x}$, the Zariski closure of the orbit in $X$ ). We say that $x=x_{s}+x_{n}$ is a Jordan decomposition for $x \in X$ if $x_{s}$ is a semisimple element with respect to $G, x_{n}$ is a nilpotent element with respect to the stabilizer subgroup of $x_{s}, G_{x_{s}}$, which is again a linear reductive group by a result of Matsuchima [Ma or Lu1], and if $G_{x}=G_{x_{s}} \cap G_{x_{n}}$ where as always $G_{y}$ denotes the stabilizer subgroup of the element $y$. Using results of Luna [Lu2], V. Kac has shown that every element $x \in X$ admits a Jordan decomposition [Ka2, p. 161].

From now on we will restrict attention to the study of the semisimple elements. Since $G$ acts linearly on $X, G$ acts as a group of automorphisms on the coordinate ring $\mathbf{C}[X]$. Because $G$ is reductive we know that the fixed ring $\mathbf{C}[X]^{G}$ for this action is affine and hence is the coordinate ring of a variety which we denote $X / G$ and call the quotient variety of $X$ under the action of $G$. The 
natural embedding $\mathbf{C}[X]^{G} \rightarrow \mathbf{C}[X]$ gives rise to a morphism $\pi: X \rightarrow X / G$ which is shown to be surjective and for each point $\xi \in X / G$ the fiber $\pi^{-1}(\xi)$ contains precisely one closed orbit [Mu]. That is, the quotient variety $X / G$ parametrizes the orbits of semisimple elements in $X$.

In general, the quotient variety $X / G$ will have lots of singularities. Still it is possible to find a nice stratification of it. Take an arbitrary point $\xi \in X / G$; then the fiber contains precisely one closed orbit which we will call $T(\xi)$ and take $x \in T(\xi)$. Then, the stabilizer subgroup $G_{x}$ is a reductive subgroup of $G$. Moreover, the conjugacy class of this subgroup depends only on the closed orbit $T(\xi)$. Conversely, if $H$ is a reductive subgroup of $G$ we can look at $(X / G)_{H}$ which we define to be the set of all points $\xi \in X / G$ such that, with notations as above, the stabilizer subgroup $G_{x}$ is conjugated in $G$ to $H$. Luna [Lu1] then proves that all these sets $(X / G)_{H}$ for $H$ a reductive subgroup of $G$ form a finite (i.e. only finitely many conjugacy classes $H$ admit a nonempty $\left.(X / G)_{H}\right)$ stratification of $X / G$ into locally closed smooth irreducible algebraic subvarieties. Moreover, the stratum $(X / G)_{H}$ lies in the closure of the stratum $(X / G)_{H^{\prime}}$ if and only if $H^{\prime}$ is conjugated to a subgroup of $H$, see also [Sw].

Next, we want to describe the local structure of the quotient variety $X / G$ near a point $\xi$. Again, let $x \in T(\xi)$ and let $N_{x}$ be the normal space to the orbit $T(\xi)$ in $x$. Then, the stabilizer subgroup $G_{x}$ acts linearly on this normal space $N_{x}$ and we can consider the quotient variety for this action $N_{x} / G_{x}$. Luna's main result then states that there is a neighborhood $V$ of 0 in $N_{x} / G_{x}$ and a neighborhood $U$ of $\xi$ in $X / G$ such that there is an étale morphism $V \rightarrow U$. In particular, this implies that the quotient variety $X / G$ near the point $\xi$ is analytically isomorphic to the (simpler) quotient variety $N_{x} / G_{x}$ near the origin.

\section{THE COORDINATE RING}

By Mumford's theory [Mu] the coordinate ring of the quotient variety $V(Q, \alpha)$ $=R(Q, \alpha) / G L(\alpha)$ is equal to the ring of polynomial invariants of the action of $G L(\alpha)$ on $R(Q, \alpha), \mathbf{C}[Q, \alpha]^{G L(\alpha)}$. In this section we will give the following description of the coordinate ring:

Theorem 1. The ring of polynomial invariants for the action of $G L(\alpha)$ on the representation space $R(Q, \alpha)$ is generated by traces of oriented cycles in the quiver $Q$ of length at most $N^{2}$ where $N=\sum_{i=1}^{n} \alpha(i)$.

Let us recall some basics from the theory of finite dimensional representations. Let $R$ be an associative algebra over $\mathbf{C}$. For every commutative $\mathbf{C}$ algebra $B$ we denote by $X_{R, N}(B)$ the set of all $\mathrm{C}$-algebra morphisms $\phi: R \rightarrow$ $M_{N}(B)$. From [Pr2, $\left.\operatorname{Pr} 3\right]$ we recall that $X_{R, N}(-)$ is a representable functor and thus we have a commutative $\mathrm{C}$-algebra $A_{R, N}$ and a universal representation $j: R \rightarrow M_{N}\left(A_{R, N}\right)$. The group $G L_{N}(\mathbf{C})$ acts functorially on the set 
$M_{N}(B)$ by conjugation and induces an action on the scheme $X_{R, N}$, an action on $A_{R, N}$ and an action on the polynomial maps $M_{N}\left(A_{R, N}\right)$ from $X_{R, N}$ to $M_{N}(\mathbf{C})$. Then, $j: R \rightarrow M_{N}\left(A_{R, N}\right)$ maps $R$ into the subring of $G L_{N}(\mathbf{C})$ equivariant maps $M_{N}\left(A_{R, N}\right)^{G L_{N}(\mathbf{C})}$. If $R$ is moreover an algebra with a trace, i.e. $\operatorname{Tr}: R \rightarrow R$ is $k$-linear satisfying $\operatorname{Tr}(a b)=\operatorname{Tr}(b a), \operatorname{Tr}(a) b=b \operatorname{Tr}(a)$ and $\operatorname{Tr}(\operatorname{Tr}(a) b)=\operatorname{Tr}(a) \operatorname{Tr}(b)$, then the map $j: R \rightarrow M_{N}\left(A_{R, N}\right)^{G L_{N}(\mathbf{C})}$ is surjective and an isomorphism if $R$ satisfies the Cayley-Hamilton polynomials for $N$ by $N$ matrices, see [Pr3].

Assume in addition that $R$ has a decomposition of 1 as a sum of orthogonal idempotents $e_{1}, e_{2}, \ldots, e_{n}$. Then we define an algebra $S_{n}=\mathbf{C}\left[e_{1}, \ldots, e_{n}\right] / J$ where $J$ is the ideal generated by the relations $e_{i}^{2}=0, e_{i} e_{j}=0$ and $\sum_{i=1}^{n} e_{i}=$ 1 ; then the scheme $X_{S_{n}, N}$ is the disjoint union of the homogeneous varieties $G L_{N}(\mathbf{C}) / G L(\alpha)$ where $\alpha$ is a dimension vector such that $\sum_{i=1}^{n} \alpha(i)=N$, i.e. $X_{S_{n}, N}=\bigcup_{\alpha} X_{\alpha}$. The inclusion $S_{n} \rightarrow R$ induces a mapping $\omega: X_{R, N} \rightarrow X_{S_{n}, N}$ so we can decompose $X_{R, N}=\bigcup_{\alpha} \omega^{-1} X_{\alpha}$. Let $A_{R, \alpha}$ be the coordinate ring of $\omega^{-1}\left(X_{\alpha}\right)$; then $A_{R, N}=\bigoplus_{\alpha} A_{R, \alpha}$ and $M_{N}\left(A_{R, N}\right)=\bigoplus_{\alpha} M_{N}\left(A_{R, \alpha}\right)$ and since $G L_{N}(\mathbf{C})$ acts separately on each summand we also have $R=\bigoplus_{\alpha} R_{\alpha}$ and the projections to $R_{\alpha}$ of the idempotent $e_{i}$ have trace $\alpha(i)$.

From now on we will restrict attention to one of these components $R_{\alpha}$. Consider the decomposition of $N$ by $N$ matrices associated to $N=\sum \alpha(i)$. Thus we decompose $1=\sum u_{i}$ where $u_{i}$ is the diagonal matrix with 1 in the positions from $\alpha(1)+\cdots+\alpha(i-1)+1$ to $\alpha(1)+\cdots+\alpha(i)$ and zeros elsewhere and for any $B, M_{N}(B)=\bigoplus_{i, j} u_{i} M_{N}(B) u_{j}$ is the corresponding block decomposition. Now, define a subfunctor $X_{R_{\alpha}}^{b}(B)=\left\{\phi: R_{\alpha} \rightarrow M_{N}(B) \mid \phi\left(e_{i}\right)=\right.$ $\left.u_{i}\right\}$. Then this subfunctor is also representable but now only the centralizer of the idempotents $u_{i}$ in $G L_{N}(\mathbf{C})$ which is equal to $G L(\alpha)$ acts on this scheme. Moreover, we claim that $R_{\alpha}$ is isomorphic to the ring of $G L(\alpha)$-equivariant maps from $X_{R_{\alpha}}$ to $M_{N}(\mathbf{C})$. For, $R_{\alpha}$ is the ring of $G L_{N}(\mathbf{C})$-equivariant maps from $X_{R_{\alpha}, N}$ to $M_{N}(\mathbf{C})$. $\omega: X_{R_{\alpha}, N} \rightarrow X_{\alpha}(\mathbf{C})$ is $G L_{N}(\mathbf{C})$-equivariant and $X_{\alpha}(\mathbf{C})$ is a homogeneous variety; the stabilizer of the point $p \in X_{\alpha(\mathrm{C})}$ corresponding to the elements $u_{i}$ is $G L(\alpha)$. Therefore, the $G L_{N}(\mathbf{C})$-equivariant maps from $X_{R_{a}, N}$ to $M_{N}(\mathbf{C})$ coincide with the $G L(\alpha)$-equivariant maps from the fiber $\omega^{-1}(p)=X_{R_{a}}^{b}$ to $M_{N}(\mathbf{C})$, finishing the proof of our claim. Further, if $A_{R_{a}}^{b}$ is the coordinate ring of $X_{R_{a}}^{b}$ then $G L(\alpha)$ acts on it and $R_{\alpha}=\left(A_{R_{\alpha}}^{b} \otimes M_{N}(\mathbf{C})\right)^{G L(\alpha)}$ and this isomorphism is compatible with the block decomposition.

Let us return to the case of interest to us. Let $\mathbf{C} Q^{0}$ be the path algebra of the opposite quiver (i.e. the quiver obtained by reversing the orientation of all the arrows of $Q)$. Then we can think of $R(Q, \alpha)$ as the variety of $N=\sum \alpha(i)$-dimensional representations of $\mathbf{C} Q^{0}$ in block form. Consider the 
algebra $T Q^{0}$ obtained from $\mathbf{C} Q^{0}$ by adding traces, imposing the relations coming from Cayley-Hamilton polynomials for $N$ by $N$ matrices and then imposing the relations $\operatorname{Tr}\left(e_{i}\right)=u_{i}$ where $e_{i}$ is the idempotent corresponding to vertex $i$. Then $R(Q, \alpha)$ is exactly the fiber $\omega^{-1}(p)$ considered above. Therefore, $T Q^{0}$ is the ring of $G L(\alpha)$-equivariant maps from $R(Q, \alpha)$ to $M_{N}(\mathbf{C})$ and interpreting further the results mentioned above we may say:

(i) The ring of $G L(\alpha)$-invariants on $R(Q, \alpha)$ (and hence the coordinate ring of the quotient variety) is generated by the traces of oriented cycles in the quiver. The bound $N^{2}$ on the length of cycles required to generate comes from the Razmyslov result [Fo].

(ii) Given two vertices $i, j$ the $G L(\alpha)$-equivariant maps from $R(Q, \alpha)$ to $\operatorname{Hom}\left(\mathbf{C}^{\alpha(i)}, \mathbf{C}^{\alpha(j)}\right)$ are generated as a module over the invariants by the paths starting from $i$ and ending in $j$.

(iii) All relations among the previously defined invariants and covariants can be deduced from the Cayley-Hamilton polynomials for $N$ by $N$ matrices.

\section{The STRATIFICATION}

In this section we will give a concrete description of Luna's general stratification result for quotient varieties in the special case of the quotient variety $V(Q, \alpha)=R(Q, \alpha) / G L(\alpha)$. The points $\xi \in V(Q, \alpha)$ are in one-to-one correspondence with the isomorphism classes of semisimple representations of $Q$ of dimension vector $\alpha$. Let $V_{\xi}$ be a semisimple representation in the fiber $\pi^{-1}(\xi)$. Then we can decompose $V_{\xi}$ in its simple components

$$
V_{\xi}=W_{1}^{\oplus e_{1}} \oplus \cdots \oplus W_{k}^{\oplus e_{k}}
$$

where $W_{i}$ is a simple representation of the quiver $Q$ of dimension vector $\beta_{i}$ which occurs in $V$ with multiplicity $e_{i}$. We will say that $\xi$ is then a point of representation type $\tau=\left(e_{1}, \beta_{1} ; \ldots ; e_{k}, \beta_{k}\right)$. Note that we will give in the next section a purely combinatorial method to describe all possible representation types. Further, with $V(Q, \alpha)_{\tau}$ we will denote the set of all points $\xi$ of $V(Q, \alpha)$ of representation type $t(\xi)=\tau$.

Theorem 2. $\left\{V(Q, \alpha)_{\tau}: \tau\right.$ a representation type $\}$ is a finite stratification of the quotient variety $V(Q, \alpha)$ into locally closed irreducible smooth subvarieties.

Proof. In view of Luna's result mentioned in $\S 2$ we have to verify that the representation type determines the stabilizer subgroup up to conjugation. So, let $\xi$ be a point of representation type $\tau=\left(e_{1}, \beta_{1} ; \ldots ; e_{k}, \beta_{k}\right)$ where $\beta_{i}=$ $\left(b_{i 1}, \ldots, b_{i n}\right) \in \mathbf{N}^{n}$ and we denote $b_{i}=\sum_{j=1}^{n} b_{i j}$. As above, let $V=\bigoplus W_{i}^{\oplus e_{i}}$ be a semisimple representation lying in the fiber $\pi^{-1}(\xi)$. Now, we can choose a basis of $\bigoplus_{i \in Q_{0}} \mathbf{C}^{\alpha(i)}$ in the following way: the first $e_{1} b_{1}$ vectors give a basis of the simple components of type $W_{1}$, the next $e_{2} b_{2}$ vectors give a basis for the simple components of type $W_{2}$ and so on. 
In this basis, the subring of $M_{N}(\mathbf{C})$ where $N=\sum \alpha(i)$ generated by the representation $V$ is

$$
\left(\begin{array}{llll}
M_{b_{1}}(\mathbf{C}) \otimes I_{e_{1}} & & \\
& \ddots & 0 & \\
& 0 & \ddots & \\
& & M_{b_{k}}(\mathbf{C}) \otimes I_{e_{k}}
\end{array}\right) .
$$

The stabilizer subgroup $G L(\alpha)_{V}$ is easily seen to be the group of units of the centralizer of this ring which is

$$
\left(\begin{array}{llll}
M_{e_{1}}\left(\mathbf{C} \otimes I_{b_{1}}\right) & & \\
& \ddots & 0 & \\
& 0 & \ddots & \\
& & M_{e_{k}}\left(\mathbf{C} \otimes I_{b_{k}}\right)
\end{array}\right) .
$$

whence $G L(\alpha)_{V} \cong G L_{e_{1}}(\mathbf{C}) \times \cdots \times G L_{e_{k}}(\mathbf{C})$ which is embedded in $G L(\alpha)$ (with respect to the particular choice of basis) as

$$
\left(\begin{array}{llll}
G L_{e_{1}}\left(\mathbf{C} \otimes I_{b_{1}}\right) & & \\
& \ddots & 0 & \\
& 0 & \ddots & \\
& & G L_{e_{k}}\left(\mathbf{C} \otimes I_{b_{k}}\right)
\end{array}\right)
$$

Now, it is fairly easy to see that the conjugacy class of $G L(\alpha)_{V}$ depends only on the representation type $\tau$, finishing the proof.

Further, one can verify that a stabilizer subgroup $G L(\alpha)_{\tau^{\prime}}$ corresponding to a representation type $\tau^{\prime}$ is conjugated to a subgroup of the stabilizer subgroup $G L(\alpha)_{\tau}$ corresponding to representation type $\tau$ if and only if $\tau^{\prime}$ is a successor of $\tau$ for the following order relation:

Two representation types

$$
\tau=\left(e_{1}, \alpha_{1} ; \ldots ; e_{k}, \alpha_{k}\right) \text { and } \tau^{\prime}=\left(e_{1}^{\prime}, \alpha_{1}^{\prime} ; \ldots ; e_{k}^{\prime}, \alpha_{k^{\prime}}^{\prime}\right)
$$

are said to be direct successors $\tau<\tau^{\prime}$ iff

(1) $k^{\prime}=k+1$ and for all but one $1 \leq i \leq k$ we have $\left(e_{i}, \alpha_{i}\right)=\left(e_{j}^{\prime}, \alpha_{j}^{\prime}\right)$ for precisely one $j$ and for the remaining $i$ we have corresponding to it $\left(e_{i}, \alpha_{l}^{\prime} ; e_{i}, \alpha_{m}^{\prime}\right)$ where $\alpha_{i}=\alpha_{l}^{\prime}+\alpha_{m}^{\prime}$, or

(2) $k^{\prime}=k-1$ and for all but one $1 \leq i \leq k^{\prime}$ we have $\left(e_{i}^{\prime}, \alpha_{i}^{\prime}\right)=\left(e_{j}, \alpha_{j}\right)$ for precisely one $j$ and for the remaining $i$ we have corresponding to it $\left(e_{l}, \alpha_{i}^{\prime} ; e_{m}, \alpha_{i}^{\prime}\right)$ where $e_{l}+e_{m}=e_{i}^{\prime}$.

Two types $\tau$ and $\tau^{\prime}$ are said to be successors $\tau \ll \tau^{\prime}$ if there exist types $\tau_{1}, \ldots, \tau_{l}$ such that $\tau=\tau_{1}<\cdots<\tau_{l}=\tau^{\prime}$. Combining this with Luna's result mentioned in $\S 2$ we get

Theorem 3. The stratum $V(Q, \alpha)_{\tau^{\prime}}$ lies in the closure of the stratum $V(Q, \alpha)_{\tau}$ if and only if $\tau \ll \tau^{\prime}$.

Therefore, Luna's stratification of the quotient variety $V(Q, \alpha)$ can be described completely by representation theoretic features. The remaining problem 
of determining which representation types can occur, which comes down to the description of the dimension vectors of simple representations, will be solved in the next section.

\section{THE SIMPLE REPRESENTATIONS}

A full subquiver $Q^{\prime}$ of $Q$ is said to be strongly connected if and only if each couple from its vertexset belongs to an oriented cycle. It is clear that we can divide $Q$ into maximal strongly connected components, say $G_{1}, \ldots, G_{k}$. The direction of all arrows between elements of $G_{i}$ and elements of $G_{j}$ is the same by the maximality condition and hence can be used to define an orientation between $G_{i}$ and $G_{j}$. The strongly connected component quiver, $S C(Q)$ of the quiver $Q$, has as its vertices the maximal strongly connected components and there is an arrow from $G_{i}$ to $G_{j}$ if and only if there is an arrow in the quiver $Q$ from an element of $G_{i}$ to an element of $G_{j}$. Remark that $S C(Q)$ is always a connected quiver without oriented cycles.

It is easy to deduce necessary conditions on the dimension vectors of simple representations. Let $V \in R(Q, \alpha)$ be a simple representation. Then we first claim that the support of $\alpha$ is a strongly connected subquiver. Assume otherwise; then we can consider the strongly connected quiver $S C(\operatorname{supp}(\alpha))$ of the support and consider a sink in it, say $H$. Then we can construct a proper subrepresentation $W$ of $V$ by

(1) For $i \in H_{0}$ let $W(i)=V(i)$ and $W(i)=0$ otherwise.

(2) For $\phi \in H_{1}$ let $W(\phi)=V(\phi)$ and $W(\phi)=0$ otherwise.

In order to state the second necessary condition, let us recall some facts about the Ringel bilinear form $R(-,-)$ on $\mathbf{Z}^{n}$ which is defined by

$$
R\left(\alpha_{i}, \alpha_{j}\right)=\delta_{i j}-r_{i j}
$$

where $r_{i j}$ is the number of directed arrows from vertex $i$ to vertex $j$ and $\alpha_{i}=\left(\delta_{i j}\right)_{j}$ are the standard basis vectors for $\mathbf{Z}^{n}$. If $V_{i} \in R\left(Q, \gamma_{i}\right)$ then we have

$$
R\left(\gamma_{1}, \gamma_{2}\right)=\operatorname{dim}_{\mathbf{C}} \operatorname{Hom}\left(V_{1}, V_{2}\right)-\operatorname{dim}_{\mathbf{C}} \operatorname{Ext}^{1}\left(V_{1}, V_{2}\right) .
$$

We now claim that for $V$ a simple representation of dimension vector $\alpha$ we have $R\left(\alpha, \alpha_{i}\right) \leq 0$ and $R\left(\alpha_{i}, \alpha\right) \leq 0$ for all $1 \leq i \leq n$. For, $R\left(\alpha_{i}, \alpha\right)=$ $\alpha(i)-\sum_{i \rightarrow j} r_{i j} \alpha(j)$ so if $R\left(\alpha_{i}, \alpha\right)>0$ then the natural morphism

$$
\bigoplus_{\phi: i \rightarrow j} V(\phi): V(i) \rightarrow \bigoplus_{\phi: i \rightarrow j} V(j)
$$

has a nontrivial kernel say $K$ which determines a proper subrepresentation $W$ of $V$ by $W(i)=K, W(j)=0$ for $j \neq i$ and $W(\phi)=0$ for all $\phi \in Q_{1}$. Similarly, if $R\left(\alpha, \alpha_{i}\right)=\alpha(i)-\sum_{j \rightarrow i} r_{j i} \alpha(j)>0$ then the image of the natural morphism

$$
\bigoplus_{\phi: j \rightarrow i}(\phi): \bigoplus_{\phi: j \rightarrow i} V(j) \rightarrow V(i)
$$


is a proper subspace of $V(i)$. Therefore, we have a proper subrepresentation $W$ of $V$ by $W(i)=\operatorname{Im}(\bigoplus V(\phi)), W(j)=V(j)$ if $j \neq i$ and $W(\phi)=V(\phi)$ for all $\phi \in Q_{1}$.

These conditions are however not sufficient to imply that $\alpha$ is the dimension vector of a simple representation. For take the extended Dynkin diagram $\tilde{A}_{n}$ with the cyclic orientation. Then $\alpha=\alpha \cdot(1, \ldots, 1)$ satisfies the conditions for all $a \in \mathbf{N}-0$. However, it is well known that the only nontrivial simple representation has dimension vector $(1, \ldots, 1)$. Nevertheless, we will now show that these are the only exceptions.

Theorem 4. $\alpha \in \mathbf{N}^{n}$ is the dimension vector of a simple representation of the quiver $Q$ if and only if either $\operatorname{supp}(\alpha)$ is the extended Dynkin diagram $\tilde{A}_{n}$ and $\alpha \mid \operatorname{supp}(\alpha)=(1, \ldots, 1)$ or $\operatorname{supp}(\alpha)$ is a noncyclic strongly connected subquiver and $B\left(\alpha, \alpha_{i}\right) \leq 0, R\left(\alpha_{i}, \alpha\right) \leq 0$ for all $1 \leq i \leq n$

The proof uses double induction both on the number $n$ of vertices in the quiver $Q$ and on $N=\sum \alpha(i)$. First, we need some extra (nonstandard) terminology: we call a vertex $i$ a focus (resp. a prism) iff there is a unique $\phi \in Q_{1}$ such that $t(\phi)=i$ (resp. $h(\phi)=i$ ). A vertex $i$ is said to be large iff $\alpha(i)$ is maximal among the $\{\alpha(j): 1 \leq j \leq n\}$. Further, we call a vertex $i$ good if and only if $i$ is large and it has no large direct successor which is a prism or a large direct predecessor which is a focus.

Lemma 1. If $Q$ is strongly connected and not the Dynkin diagram $A_{n}$, then there does not exist a cycle of prism (resp. focus) vertices.

Proof. Suppose there is a cycle of prisms $\left(i_{1}, \ldots, i_{k}\right)$. Then for each $1 \leq j \leq k$ the unique arrow coming into $i_{j}$ belongs to the cycle. However, $Q$ itself is not a cycle so there is at least one extra vertex $i$. But, there is no path from $i$ to any of the $i_{j}$ contradicting strong connectness of $Q$.

Using this lemma we can find either a good vertex or a large prism $i$ which has no large prism direct successors. If we are in the second case, then the unique predecessor $j$ of $i$ has to be a large focus and we can apply a shrinking process:

Lemma 2. If we have a prism vertex $i$ with unique predecessor a focus vertex $j$ and $\alpha(i)=\alpha(j)$ then $\alpha$ is the dimension vector of a simple representation of $Q$ if and only if $\alpha^{\prime}=(\alpha(1), \ldots, \alpha(i-1), \alpha(i+1), \ldots, \alpha(n)) \in \mathbf{N}^{n-1}$ is the dimension vector of a simple representation of the quiver $Q^{\prime}$ obtained from $Q$ by identifying the vertices $i$ and $j$

Proof. If $\phi$ is the unique arrow from $j$ to $i$ and if $V$ is a simple representation with dimension vector $\alpha$ then it is easy to see that $V(\phi)$ is an isomorphism, so we can identify $V(i)$ with $V(j)$ and obtain a simple representation of $Q^{\prime}$.

Conversely, if $V^{\prime}$ is a simple representation of $Q^{\prime}$, then we can form a representation $V$ of $Q$ such that $V^{\prime}(k)=V(k)$ for all $k \neq i$ and $V(i)=$ $V^{\prime}(j), V(\phi)=I_{V^{\prime}(j)}$ and all other morphisms are the ones from $V^{\prime}$. Then it is easy to check that $V$ is indeed a simple representation. 
The foregoing lemma finishes the proof of Theorem 4 in case we do not have a good vertex by induction on the number of vertices (note that the Ringel form condition is preserved in passing from $Q$ to $Q^{\prime}$ by the fact that $\left.\alpha(i)=\alpha(j)\right)$. Therefore, we are left to consider the case in which there exists a good vertex $i$. If $\alpha(i)=1$ then for all $j \in \operatorname{supp}(\alpha)$ we have $\alpha(j)=1$. Then, if we put for all $V(\phi)=I_{\mathrm{C}}$ we get a simple representaton $V$ because $\operatorname{supp}(\alpha)$ is supposed to be strongly connected.

If $\alpha(i)>1$ then we replace the dimension vector $\alpha$ by $\alpha^{\prime}$ where $\alpha^{\prime}(j)=\alpha(j)$ for all $j \neq i$ and $\alpha^{\prime}(i)=\alpha(i)=1$. Clearly $\operatorname{supp}\left(\alpha^{\prime}\right)=\operatorname{supp}(\alpha)$ so it is strongly connected and we claim that still $R\left(\alpha^{\prime}, \alpha_{j}\right) \leq 0$ and $R\left(\alpha_{j}, \alpha\right) \leq 0$ for all $1 \leq j \leq n$. The only possible vertices $j$ where things might go wrong are direct predecessors and direct successors of $i$. Suppose for one of them $R\left(\alpha_{j}, \alpha^{\prime}\right)>0$. Then $\alpha^{\prime}(j)>\sum_{j \rightarrow k} r_{j k} \alpha^{\prime}(k) \geq \alpha^{\prime}(i)$ whence $\alpha^{\prime}(j)=\alpha(j)=\alpha(i)$ whence $j$ must be a large vertex and a focus with end point $i$, contradicting the goodness of vertex $i$.

So, by induction on $N$ we may assume that there exists a simple representation of the quiver $Q$ of dimension vector $\alpha^{\prime}$. Take such a representation $V^{\prime} \in R\left(Q, \alpha^{\prime}\right)$. Then since $R\left(\alpha^{\prime}, \alpha_{i}\right)<0$ and $R\left(\alpha_{i}, \alpha^{\prime}\right)<0$ we know that $\operatorname{Ext}^{1}\left(V^{\prime}, S_{i}\right) \neq 0 \neq \operatorname{Ext}^{1}\left(S_{i}, V^{\prime}\right)$ where $S_{i}$ is the trivial simple representation in vertex $i$. Now, look at the space of all representations $V \in R(Q, \alpha)$ having the property that $V \mid \alpha^{\prime}=V^{\prime}$. This is an affine space $X_{V^{\prime}}$ of dimension $\sum_{j \rightarrow i} r j i \alpha^{\prime}(j)+\sum_{i \rightarrow j} r_{i j} \alpha^{\prime}(j)$. Loosely speaking, $X_{V^{\prime}}$ consists of those representations which are worse than $V^{\prime} \oplus S_{i}$. We can choose the representation $V^{\prime}$ in such a way that $X_{V^{\prime}}$, contains representations with a trace of an oriented cycle different from the corresponding trace of $V^{\prime} \oplus S_{i}$. This can be done because being simple is an open condition in $R\left(Q, \alpha^{\prime}\right)$. Therefore, the Jordan-Hölder factors of these representations cannot be $V^{\prime}$ and $S_{i}$ (see $\S 3$ ) but still they degenerate to $V^{\prime} \oplus S_{i}$; hence by the stratification result they must be simple, finishing the proof of Theorem 4.

Recall that a representation $V$ of a quiver $Q$ is called a Schur representation if its endomorphism ring is $\mathbf{C}$; the dimension vector of a Schur representation is called a Schur root. V. Kac conjectured a purely combinatorial description of these Schur roots in [Ka2]. He defines a vector $\alpha \in \mathbf{N}^{n}$ to be indecomposable if $\alpha$ cannot be written as a sum $\beta+\gamma$ with $R(\beta, \gamma) \geq 0$ and $R(\gamma, \beta) \geq 0$. He then conjectured that Schur roots and indecomposable vectors coincide. In general, this conjecture is false, see [LB]. However, it is clear from [KR, Lemma 3.2] and Theorem 4 that the Schur roots which are dimension vectors of simple representations are indeed indecomposable.

\section{THE LOCAL STRUCTURE}

In this section we will apply Luna's étale slice theorem (see $\S 2$ ) in order to study the étale local structure of the quotient variety $V(Q, \alpha)=R(Q, \alpha) / G L(\alpha)$. 
Suppose that $\xi \in V(Q, \alpha)$ is of representation type $\tau=\left(e_{1}, \beta_{1} ; \ldots ; e_{k}, \beta_{k}\right)$. We will construct a new quiver $Q_{\tau}$ in the following way: $\left(Q_{\tau}\right)_{0}=\{1, \ldots, k\}$ and there are precisely $\delta_{i j}-R\left(\beta_{i}, \beta_{j}\right)$ directed arrows from $i$ to $j$. Consider the dimension vector $\alpha_{\tau}=\left(e_{1} ; \ldots ; e_{k}\right) \in \mathbf{N}^{k}$. Then we will prove the following result

Theorem 5. If $\xi \in V(Q, \alpha)$ is of representation type $\tau$, then (with notations as above) there is an étale morphism from a neighborhood of the origin in the quotient variety $V\left(Q_{\tau}, \alpha_{\tau}\right)$ to a neighborhood of $\xi$ in $V(Q, \alpha)$.

In view of the Luna slice theorem, it suffices to show that the normal space to the orbit of a semisimple representation $V$ corresponding to $\xi$ is isomorphic as a $G L(\alpha)_{V}$-representation to the representation space $R\left(Q_{\tau}, \alpha_{\tau}\right)$ (note that we have shown in $\S 4$ that the stabilizer subgroup in $V$ is isomorphic to $\left.G L\left(\alpha_{\tau}\right)\right)$. We know that the tangentspace to the $G L(\alpha)$-orbit in $V$ is equal to the image of the natural linear map

$$
\operatorname{Lie}(G L(\alpha)) \rightarrow R(Q, \alpha)
$$

sending an element $y \in \operatorname{Lie}(G L(\alpha))$ to the representation determined by the commutator $[y, V]=y \cdot V-V \cdot y$, that is each $V(\phi)$ can be extended with zero blocks to an $N$ by $N$ matrix which we call $W(\phi)$ and then $[y, V](\phi)=$ $[y, W(\phi)] \in M_{N}(\mathbf{C})$. The kernel of this map is clearly the centralizer of the subalgebra of $M_{N}(\mathbf{C})$ generated by the representation $V$ (i.e. by the matrices $W(\phi)$ mentioned above).

Let us choose a basis of $\mathbf{C}^{N}$ as in the proof of Theorem 2. Then this centralizer is

$$
C_{V}=\left(\begin{array}{ccc}
M_{e_{1}}\left(\mathbf{C} \otimes I_{b_{1}}\right) & 0 \\
0 & \ddots & M_{e_{k}}\left(\mathbf{C} \otimes I_{b_{k}}\right)
\end{array}\right)
$$

and therefore we obtain an exact sequence of $G L(\alpha)_{V}$-modules

$$
0 \rightarrow C_{V} \rightarrow \operatorname{Lie}(G L(\alpha)) \rightarrow T_{V}\left(G L(\alpha)_{V}\right) \rightarrow 0
$$

where the action of $G L(\alpha)_{V}$ is of course by conjugation in $M_{N}(\mathbf{C})$. Note also that $\operatorname{Lie}(G L(\alpha))$ has to be viewed as a subalgebra of $M_{N}(\mathbf{C})$ depending on the particular choice of basis (which complicates the computations somewhat). A typical element $\gamma \in G L(\alpha)_{V} \cong G L\left(\alpha_{\tau}\right)=\prod_{i=1}^{k} G L_{e_{i}}(\mathbf{C})$ will be written as $\left(\gamma_{1}, \ldots, \gamma_{k}\right)$ and the actions will be expressed in terms of the $\gamma_{i}$ 's. It is easy to see that $C_{V}$ consists of

$1 e_{1}^{2}$-dimensional representation with $\gamma_{1}^{-1} \cdot \gamma_{1}$-action,

$1 e_{2}^{2}$-dimensional representation with $\gamma_{2}^{-1} \cdot \gamma_{2}$-action,

$1 e_{k}^{2}$-dimensional representation with $\gamma_{k}^{-1} \cdot \gamma_{k}$-action.

If we recall our notation that $\beta_{i}=\left(b_{i 1}, \ldots, b_{i n}\right)$ then one can verify that 
$\operatorname{Lie}(G L(\alpha))$ consists of

$\sum_{j=1}^{n} b_{1 j}^{2} e_{1}^{2}$-dimensional representations with $\gamma_{1}^{-1} \cdot \gamma_{1}$-action,

$\sum_{j=1}^{n} b_{2 j}^{2} e_{2}^{2}$-dimensional representations with $\gamma_{2}^{-1} \cdot \gamma_{2}$-action,

$\sum_{j=1}^{n} b_{k j}^{2} e_{k}^{2}$-dimensional representations with $\gamma_{k}^{-1} \cdot \gamma_{k}$-action. Moreover, there are

$\sum_{j=1}^{n} b_{1 j} b_{2 j} e_{1} \times e_{2}$-dimensional representations with $\gamma_{1}^{-1} \cdot \gamma_{2}$-action,

$\sum_{j=1}^{n} b_{k j} b_{k-1 j} e_{k} \times e_{k-1}$-dimensional representations with $\gamma_{k}^{-1} \cdot \gamma_{k-1}$-action. From these descriptions and the exact sequence it is then easy to give a full description of $T_{V}(G L(\alpha) V)$ as a $G L(\alpha)_{V}$-module. Next, the normal space to the orbit and its $G L(\alpha)_{V}$-action is determined by the exact sequence of $G L(\alpha)_{V^{-}}$ modules

$$
0 \rightarrow T_{V}(G L(\alpha) V) \rightarrow R(Q, \alpha) \rightarrow N_{V} \rightarrow 0 .
$$

In order to complete the proof we have to give a detailed description of the action of $G L(\alpha)_{V}$ on the representation space $R(Q, \alpha)$. One can verify that the part of $R(Q, \alpha)$ corresponding to a directed arrow $\phi$ from vertex $i$ to vertex $j$ consists of

$b_{1 i} b_{1 j} e_{1} \times e_{1}$-dimensional representations with $\gamma_{1}^{-1} \cdot \gamma_{1}$-action,

$b_{1 i} b_{2 j} e_{1} \times e_{2}$-dimensional representations with $\gamma_{1}^{-1} \cdot \gamma_{2}$-action,

$b_{k i} b_{k j} e_{k} \times e_{k}$-dimensional representations with $\gamma_{k}^{-1} \cdot \gamma_{k}$-action.

Repeating this for every arrow $\phi \in Q_{1}$ we get a full description of the $G L(\alpha)_{V^{-}}$ module structure of $R(Q, \alpha)$ and hence using the exact sequence and the description obtained above of $T_{V}(G L(\alpha) V)$ we get the $G L(\alpha)_{V}$-module structure of the normalspace $N_{V}$. We leave it to the reader to check that this action coincides with the natural action of $G L\left(\alpha_{\tau}\right) \cong G L(\alpha)_{V}$ on the representation space $R\left(Q_{\tau}, \alpha_{\tau}\right)$.

\section{ODDS AND ENDS}

In view of the stratification result (Theorem 2 ) there is precisely one semisimple representation type $\tau_{g}$ such that the corresponding stratum $V(Q, \alpha)_{\tau_{g}}$ is an open subvariety of the quotient variety $V(Q, \alpha)$. We call $\tau_{g}$ the generic representation type. We will now indicate how it can be determined: given the quiver $Q$ and the dimension vector $\alpha$ we can consider as before the strongly connected component quiver $S C(\operatorname{supp}(\alpha))$. A simple subrepresentation of a generic representation in $R(Q, \alpha)$ must live on one of the sinks of $S C(\operatorname{supp}(\alpha))$. So, restrict attention to one of these strongly connected components. Then there is exactly one maximal dimension vector $\beta \leq \alpha$ living on it such that $R\left(\beta, \alpha_{i}\right) \leq 0$ and $R\left(\alpha_{i}, \beta\right) \leq 0$ for all $i$. The generic representation type on this strongly connected component is then easily seen to be

$$
\left(1, \beta ; \alpha\left(i_{1}\right)-\beta\left(i_{1}\right), \alpha_{i_{1}} ; \ldots ; \alpha\left(i_{j}\right)-\beta\left(i_{j}\right), \alpha_{i_{j}}\right)
$$


if $\left\{i_{1}, \ldots, i_{j}\right\}$ are the vertices in the strongly connected component. Having determined the generic representation type in one of the sinks of the strongly connected component quiver, we delete this sink from it and repeat the above procedure until we reach the empty graph. The generic representation type $\tau_{g}$ will then be the sum of the generic types of the maximal strongly connected components. So, there is a purely combinatorial procedure to determine the generic representation type

$$
\tau_{g}=\left(e_{1}, \gamma_{1} ; \ldots ; e_{l}, \gamma_{l}\right) .
$$

Now, consider the étale local structure in a point of generic representaiton type. In view of the structure of the strongly connected component quiver $S C(\operatorname{supp}(\alpha))$ one can verify that the quiver $Q_{\tau_{g}}$ has no oriented cycles other than loops occurring in vertices $i$ such that $\alpha_{\tau_{g}}(i)=1$ (corresponding to the nontrivial simples living in the maximal strongly connected components). So, combining Theorems 1 and 5 we see that the coordinate ring of $V\left(Q_{\tau_{g}}, \alpha_{\tau_{g}}\right)$ is a polynomial ring in as many variables as there are loops in such vertices. Hence we have proved

Theorem 6. If $\tau_{g}=\left(e_{1}, \gamma_{1} ; \ldots ; e_{l}, \gamma_{l}\right)$ is the generic (semisimple) representation type, then the quotient variety $V(Q, \alpha)$ has dimension $\sum_{i=1}^{l}\left(1-R\left(\gamma_{i}, \gamma_{i}\right)\right)$.

From the above discussion it follows also that points of generic representation type are smooth points on $V(Q, \alpha)$. Moreover, in the quivers $Q_{\tau}$ corresponding to nongeneric representation types there are always oriented cycles. So, if all dimensions $\alpha(i)$ are sufficiently large, they will lead to extra relations between the generators of the coordinate ring of $V\left(Q_{\tau}, \alpha_{\tau}\right)$ so it cannot be a polynomial ring. In view of the étale local structure result this proves

Theorem 7. Except for low dimensional anomalies, the singular locus of the quotient variety $V(Q, \alpha)$ coincides with the complement of the generic stratum $V(Q, \alpha)_{\tau_{g}}$

Let us give an example of an exceptional case: consider the quiver on two vertices with corresponding Ringel form determined by the matrix

$$
\left(\begin{array}{cc}
1 & -2 \\
-1 & 1
\end{array}\right)
$$

and consider a dimension vector $\alpha=(n, 2)$ with $n \geq 2$. Then the generic type is $(1,(2,2) ; n-2,(1,0))$. Now, consider the nongeneric type $\tau=(1,(1,1) ; 1$, $(1,1) ; n-2,(1,0))$ Then the quiver $Q_{\tau}$ has its Ringel form determined by the matrix

$$
\left(\begin{array}{lll}
-1 & -1 & 0 \\
-1 & -1 & 0 \\
-1 & -1 & 1
\end{array}\right)
$$

and the corresponding dimension vector is $\alpha_{\tau}=(1,1, n-2)$, so the coordinate ring of the quotient variety $V\left(Q_{\tau}, \alpha_{\tau}\right)$ is a polynomial ring in 5 variables (4 
coming from the loops and 1 from the only extra oriented cycle). So, points of type $\tau$ are also smooth points of $V(Q, \alpha)$; in fact it can be shown that $V(Q, \alpha)$ is affine 5 -space. However, if we replace the dimension vector $\alpha$ by $\alpha^{\prime}=(n, 3)$ with $n>3$ one can show that the singular locus of $V\left(Q, \alpha^{\prime}\right)$ coincides with the complement of the generic stratum.

\section{REFERENCES}

[Fo] E. Formanek, Generating the ring of matrixinvariants, Lecture Notes in Math., vol. 1197, Springer-Verlag, Berlin and New York, 1986, pp. 73-82.

[Ga] P. Gabriel, Unzerlegbare Darstellungen. I, Manuscripta Math. 6 (1972), 71-103.

[Ka] V. Kac, Infinite root systems, representations of graphs and invariant theory, Invent. Math. 56 (1980), 57-92.

[Ka2] _ _ Infinite root systems, representations of graphs and invariant theory. II, J. Algebra 78 (1982), 141-162.

[KR] H. Kraft and Chr Riedtmann, Geometry of representations of quivers (to appear).

[Lb] L. Le Bruyn, Counterexamples to the Kac conjecture on Schur roots, Bull. Sci. Math. 110 (1986), 437-448.

[Lu1] D. Luna, Slices étales, Bull. Soc. Math. France Mém. 33 (1973), 81-105.

[Lu2] __, Adhérences d'orbites et invariants, Invent. Math. 29 (1975), 231-238.

[Ma] Y. Matsuchima, Espaces homogènes de Stein des groupes de Lie complexes, Nagoya Math J. 16 (1960), 205-218.

[Mu] D. Mumford, Geometric invariant theory, Springer-Verlag, Berlin and New York, 1965.

[Pr2] C. Procesi, Rings with polynomial identities, Marcel Dekker, 1973.

[Pr3] _ A formal inverse to the Cayley-Hamilton theorem, J. Algebra 107 (1987), 63-74.

[Sc] A. Schofield, Generic representations of quivers (to appear).

[Sw] G. Schwartz, Lifting smooth homotopies of orbit spaces, Publ. Math. Inst. Hautes Études Sci. (to appear).

Department of Mathematics, University of ANTwerp, Antwerp, Belgium

Dipartimento di Matematica, Istituto Guido Castelnuovo, Roma, Italy 